\title{
Product clustering as a strategy for enhanced plastics recycling from WEEE
}

\author{
J.R. Duflou(1) ${ }^{1, *}$, A. Boudewijn ${ }^{1}$, D. Cattrysse ${ }^{1}$, F. Wagner ${ }^{1}$, A. Accili², G. Dimitrova ${ }^{3}$, J.R. Peeters ${ }^{1}$ \\ 1 Department of Mechanical Engineering, KU Leuven, Belgium, Member of Flanders Make \\ 2 Ecodom, Lainate (Milan), Italy \\ 3 Fraunhofer Institute for Reliability and Microintegration IZM, Berlin, Germany
}

\begin{abstract}
The ambition to extend material recycling from end-of-life electric and electronic products seems to lead to an unavoidable trade-off between circularity maximisation and quality concerns. However, optimized pre-treatment can help to assure that both polymer fraction compatibility and imperfect separation with the available technologies are fully taken into account to assure maximum plastics recycling. A systematic approach to determine pre-sorting product and component clusters, that allow recycling with conventional separation techniques, is explained. Using statistical data sets for a case study of selected common WEEE products categories, the clusters in which to sort the products and dismantled components are documented and can support enhanced polymer recycling in an industrial setting.
\end{abstract}

Recycling; Polymer; Clustering strategies

\section{Introduction}

In 2016 globally 44,7 million tonnes of waste of electrical and electronic equipment (WEEE) had to be processed and, with an estimated annual growth rate of 3 to $5 \%$, this constitutes one of the fastest growing waste streams [1]. Depending on the category of products, the amount of plastics in WEEE ranges from 15 to 30 wt\% [2-4]. The plastic content in WEEE has a substantial raw material value [1]. Not only the potential economic value provides a driver to optimize the end-of-life (EoL) scenario for polymer containing WEEE streams: also more demanding governmental regulations stimulate innovation in the treatment of end-of-life electric and electronic devices. In the EU the WEEE directive recast imposes ambitious recovery, reuse and recycling requirements, causing also the plastic fractions in different categories of devices to become explicit targets [5]. For example, from 15 August 2018 onwards at least $80 \%$ of the materials in white good products are to be reused or recycled, a requirement that can hardly be met without recycling a substantial fraction of the contained plastics.

However, while PP, PC, PE, ABS and HIPS represent the biggest shares of polymers in WEEE [6], the common use of additives, such as pigments, fire retardants (FRs) and fillers, results in a huge diversity of plastics, tailored to the technical requirements imposed by specific applications. This makes systematic retrieval of Post-Consumer Recycled (PCR) plastics from WEEE all but obvious. Furthermore, the historic use of substances, such as Brominated FRs (BrFRs), some of which are classified as Persistent Organic Pollutants (POPs), has resulted in the demand for strict risk containment when dealing with PCR plastics. In this context the admissible content of Deca-BDE or PBDEs in PCR plastics has recently been under debate. In the latest recast of the EU POP regulation the combined allowed level of PBDEs in mixed plastics is $500 \mathrm{ppm}$, with a possible review foreseen for 2021 [7]. Some exceptions are specified, such as the application in electronic and electrical appliances with a RoHS directive threshold of $1000 \mathrm{ppm}$.

\section{Problem Statement}

The traditional practice in end-of-life treatment centres for WEEE consists of destructive separation by shredding and density based sorting, after magnetic and eddy current based removal of the metal fractions. However, compliance with both the WEEE and POPs regulations [5,7] requires more refined sorting of plastics, exceeding the retrieval levels that can be achieved with density based sorting only [8]. In this context the use of X-Ray Transmission (XRT), X-Ray Fluorescence (XRF) and potentially Laser-Induced Breakdown Spectroscopy (LIBS) can ultimately lead to effective separation of materials containing brominated flame retardants, regardless of colour concerns. For EoL product streams treated in a dedicated dismantling line such an approach has proven effective [9]. However, sensing based sorting techniques require significant investments and are to date not broadly implemented for large, varied streams of WEEE plastics. Therefore, in this contribution an alternative approach is considered, based on strategic pre-sorting of different categories of WEEE products, taking into account statistic information with respect to the polymers they contain and compatible with commonly available density separation facilities. This pre-sorting strategy can be complementary to state-of-the-art sensing based plastics sorting techniques as they become available. The optimisation methods underlying the proposed pre-sorting strategy are presented in this contribution and their effectiveness in increasing the fraction of recycled plastics is illustrated by means of case study data. 


\section{Product Clustering Strategy}

Besides as part of an economic value optimisation concern, separation of different plastics fractions can be desirable for the following reasons:

- Incompatibility of the containing polymers in terms of resulting properties of blended plastics results in nonrecyclable combinations of plastics. For polymer miscibility ordinal data are available among others in [10].

- $\quad \mathrm{REACH}$ and ROHS regulations: plastics fractions possibly containing restricted substances cannot be recycled and require separation and high temperature combustion in order to be compliant: e.g. [7].

\subsection{Data collection}

In order to determine the need for separation, the composition of different product categories as listed in Table 1 was studied by means of an extensive sampling campaign, taking into account separate components as can easily be distinguished by WEEE materials recycling centres. Data regarding TV and monitor back covers were available from a previous study [11]. The relative quantities of different categories were obtained through mass flow analysis.

Table 1 Product categories and their components with number of samples analysed, observed polymer types and relative weight fraction per category

\begin{tabular}{|l|c|l|r|}
\hline Product / component & \# samples & Containing polym. & Wt\% \\
\hline LCD TV back cover & 777 & $1,2,4-6,8,9$ & 10,2 \\
CRT TV back cover & 384 & $1-3,5-10$ & 10,2 \\
LCD monitor back cover & 158 & $1-5,9$ & 10,2 \\
CRT monitor back cover & 146 & $1-3,5,6,8,9$ & 10,2 \\
\hline Freezer interior & 49 & 1,5 & 3,4 \\
Refrigerator interior & 39 & 5 & 13,6 \\
Refrigerator drawer & 39 & 5 & 5,1 \\
Refrigerator door & 20 & 5 & 6,7 \\
\hline Dishwasher front panel & 20 & 1 & 10,2 \\
Washing mach.front cover & 20 & 1,9 & 6,7 \\
Washing machine tub & 20 & 10 & 13,6 \\
\hline
\end{tabular}

1: ABS; 2: ABS BrFR; 3: ABS PFR 4: ABS/PMMA; 5: HIPS; 6: HIPS BrFR

7: PA; 8: PC/ABS 9: PC/ABS PFR; 10: PP

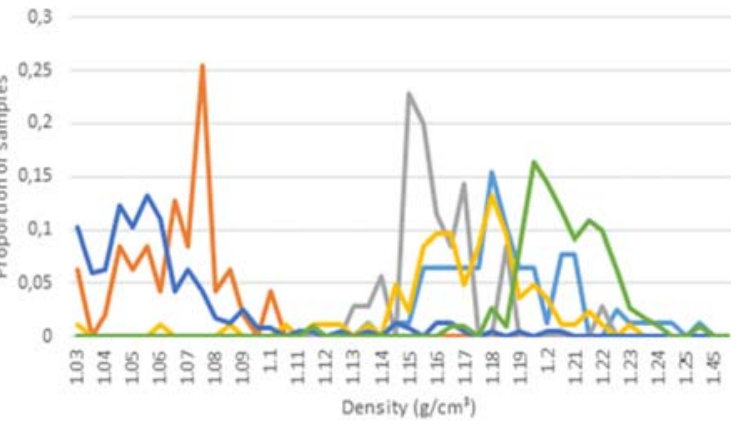

-ABS BrFR $\longrightarrow$ ABS $\longrightarrow$ ABS/PMMA — HIPS BrFR - HIPS $\longrightarrow$ PC/ABS PFR

Figure 1. Density spectra for plastics based on different polymers as contained in the product categories included in Table 1, excluding PP for which literature based density information was used.

To analyse the polymeric and flame retardants composition, back cover housings of monitors and TVs were measured with a Quantom ${ }^{\circledR}$ LIBS system of Bertin Technoplogies in an industrial trial. For the other considered devices, discs were cut out of selected components and the plastic composition was measured by Attenuated Total Reflectance FTIR using a Thermo Scientific ${ }^{\mathrm{TM}}$
Nicolet $^{\mathrm{TM}}$ iS ${ }^{\mathrm{TM}} 5$ Spectrometer. The measurement results were obtained by matching obtained spectra to a reference database of WEEE plastics using the Thermo Scientific ${ }^{\text {TM }}$ OMNIC ${ }^{\text {TM }} 9$ software package. A float bath was used to measure material densities of 1672 plastic samples. Colours were manually documented based on visual inspection.

As can be observed in Figure 1, the use of a variety of additives results in rather broad and overlapping density distributions for the distinguished plastics categories.

\subsection{Separability modelling}

In consequence of the overlapping density spectra, plastics cannot always be sorted effectively based on density separation only. In order to quantify the need for additional sorting measures, the proportion of overlap between pairs of normalized density spectra can be computed as an indicator $I$ : see Fig. 2.

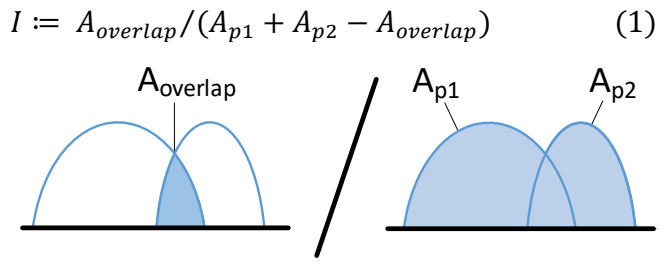

Figure 2. Overlap indicator quantifying the difficulty to automatically sort plastics fractions for two plastics $\mathrm{p} 1$ and $\mathrm{p} 2$ based on density separation

Table 2 Overlap Indicator values for normalized density spectra

\begin{tabular}{|l|c|c|c|c|c|c|c|c|c|c|}
\hline & $\mathbf{1}$ & $\mathbf{2}$ & $\mathbf{3}$ & $\mathbf{4}$ & $\mathbf{5}$ & $\mathbf{6}$ & $\mathbf{7}$ & $\mathbf{8}$ & $\mathbf{9}$ & $\mathbf{1 0}$ \\
\hline 1. ABS & 1,00 & & & & & & & & & \\
\hline 2. ABS BrFR & 0,00 & 1,00 & & & & & & & & \\
\hline 3. ABS PFR & 0,00 & 0,35 & 1,00 & & & & & & & \\
\hline 4. ABS/PMMA & 0,00 & 0,23 & 0,04 & 1,00 & & & & & & \\
\hline 5. HIPS & 0,40 & 0,04 & 0,01 & 0,02 & 1,00 & & & & & \\
\hline 6. HIPS BrFR & 0,02 & 0,54 & 0,24 & 0,29 & 0,06 & 1,00 & & & & \\
\hline 7. PA & 0,00 & 0,06 & 0,00 & 0,15 & 0,03 & 0,11 & 1,00 & & & \\
\hline 8. PC/ABS & 0,15 & 0,00 & 0,00 & 0,00 & 0,25 & 0,01 & 0,00 & 1,00 & & \\
\hline 9. PC/ABS PFR & 0,00 & 0,26 & 0,50 & 0,03 & 0,02 & 0,15 & 0,00 & 0,00 & 1,00 & \\
\hline 10. PP & 0,00 & 0,00 & 0,00 & 0,00 & 0,00 & 0,00 & 0,00 & 0,23 & 0,00 & 1,00 \\
\hline
\end{tabular}

Using Overlap Indicator values as listed in Table 2, a graph representation can be defined in which vertices are used to represent the different distinguished categories of plastics. The largest fraction of overlap between the density spectra of two plastics that can be permitted is referred to as threshold $\tau$. An edge is defined between the vertices of any two plastics if, and only if, the overlap of the associated plastics I does not exceed $\tau$. An example of the construction of this graph is shown in Fig. 3 for three hypothetical plastics A, B and C.
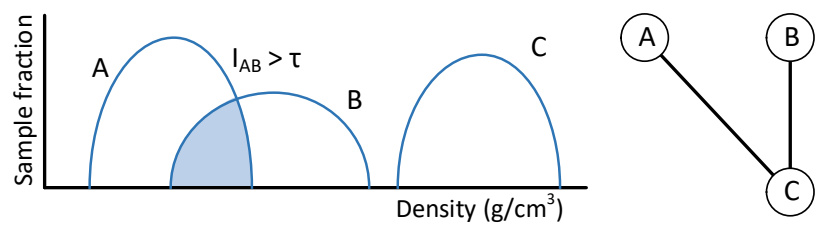

Figure 3. Construction of a graph representation (right) based on the overlap fraction of density spectra for plastics categories (left)

Since plastics connected by an edge in the graph can be separated using density-based methods, the aim is to decompose the graph into subgraphs such that there is an edge between any pair of vertices in any subgraph. For the example in Fig. 3, three such decompositions exist, namely $\{\{\mathrm{A}, \mathrm{C}\},\{\mathrm{B}\}\} ;\{\{\mathrm{A}\},\{\mathrm{B}, \mathrm{C}\}\}$ and $\{\{A\},\{B\},\{C\}\})$. The decompositions satisfying this property 
assign each plastic to a class in which all plastics can be isolated using density based sorting. Such a decomposition is most efficient if it contains as few subgraphs as possible since this requires the least effort for pre-sorting products and components. It turns out that the problem of identifying this graph decomposition is the dual of the so-called vertex colouring problem, a well-known problem in graph theory [12]. When solving this problem for the given Overlap Indicator values of Table 2 with $\tau=0,2$, five classes of polymers are obtained (see Fig. 4).

This methodology can also be applied to polymer miscibility. If instead of working directly with the graphs for density-based sorting and miscibility individually, the union of their vertex and edge sets are used, then both considerations can be taken into account simultaneously.

\subsection{Product and component clustering}

Once the graph decomposition is computed, the sum of the concentrations of plastics present in a product or component and allocated to a specific subgraph (class) can be used as an indicator for class membership. In this manner, the subgraphs can be converted into features. Thus, suppose $\mathrm{C}$ is a product component and graph $\mathrm{H}$ is one of the obtained subgraphs of the graph of materials. Let $\mathrm{P}(\mathrm{H})$ denote the set of plastics corresponding to the vertices in $\mathrm{H}$. Finally, for a given polymer $\mathrm{x}$, let $[\mathrm{x}]_{\mathrm{C}}$ denote its proportion in component $\mathrm{C}$. Then we can define the feature Feat ${ }_{H}$ on $\mathrm{C}$ through Formula (2).

$$
\text { Feat }_{H}(C):=\sum_{x \in P(H)}[x]_{C}
$$

Thus, Feat $_{H}(C) \in[0,1]$ represents what weight fraction of component $\mathrm{C}$ is present in class of polymers $\mathrm{H}$.

The method can be further extended with colour information if sorting of plastics according to specific colours is envisaged. Since a limited fraction of dark plastics can destroy the possibility to create a white or light coloured blend, separation of light coloured plastics may be desirable. For this purpose in Figure 4 an elementary binary colour characterisation as used in the case study has been added per product and component category.

\begin{tabular}{|c|c|c|c|c|c|c|c|c|}
\hline Product and component & Feat_0 & Feat_1 & Feat_2 & Feat_3 & Feat_4 & White & Colour & Cluster \\
\hline CRT Monitors back cover & 0,700 & 0,063 & 0,008 & 0,039 & 0,160 & 0,063 & 0,937 & 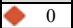 \\
\hline CRT TVs back cover & 0,031 & 0,078 & 0,052 & 0,747 & 0,018 & 0,000 & 1,000 & 0 \\
\hline Dishwashers front panel & 0,000 & 0,920 & 0,000 & 0,000 & 0,000 & 0,692 & 0,308 & 1 \\
\hline Freezers interior & 0,000 & 0,080 & 0,000 & 0,920 & 0,000 & 1,000 & 0,000 & O \\
\hline LCD Monitors back cover & 0,006 & 0,611 & 0,000 & 0,228 & 0,150 & 0,026 & 0,974 & $\Delta$ \\
\hline LCD TVs Back Cover & 0,041 & 0,124 & 0,130 & 0,370 & 0,240 & 0,008 & 0,992 & c \\
\hline Refrigerators drawers & 0,000 & 0,000 & 0,000 & 0,950 & 0,000 & 1,000 & 0,000 & 2 \\
\hline Refrigerators door & 0,000 & 0,000 & 0,000 & 1,000 & 0,000 & 1,000 & 0,000 & 2 \\
\hline Refrigerators interior & 0,000 & 0,000 & 0,000 & 0,950 & 0,000 & 1,000 & 0,000 & 2 \\
\hline Washing machines tub & 0,000 & 1,000 & 0,000 & 0,000 & 0,000 & 0,100 & 0,900 & 1 \\
\hline Washing machines front cover & 0,000 & 0,950 & 0,000 & 0,000 & 0,050 & 0,853 & 0,147 & 1 \\
\hline
\end{tabular}

Class 0: $\{$ ABS BrFR, PC/ABS $\}$, Class 1: $\{$ ABS/PMMA, ABS, PP $\}$, Class 2: \{HIPS BrFR $\}$, Class 3: \{HIPS, ABS PFR $\},$ Class 4: $\{$ PC/ABS PFR,PA\}

Figure 4. Feature class and colour class membership heat map for the case study products and components categories

Using the vectorisation facilitated by the feature values according to Formula 2, as visualised in Fig. 4, clustering of products or components is now possible based on Euclidian distance calculation.

\section{Results}

Using k-Means clustering on the dataset summarised in Table 1 and Figure 1, the selected products and components were grouped for different predefined numbers of clusters and based on both plastics composition and colour information. Taking into account the cost for combustion of non-recyclable plastic fractions and for density based sorting (costs per unit mass), and using 2019 average market values in the EU for sufficiently pure fractions or useful blends as obtained from industrial partners, the resulting return of plastics recycling as a function of the number of distinguished product clusters was quantified as shown in Fig. 5. In this exercise the cost for sorting products according to the obtained cluster results was neglected. This costs is expected to be modest if logistic systems for product collection can be adjusted to clustering prescriptions. However, a cost per distinguished cluster can be anticipated since separate storage of product clusters and emptying of shredder facilities between processing of different clusters will be required. Based on these considerations three clusters was determined to be the advisable number for pre-sorting the considered products and components in this case study.

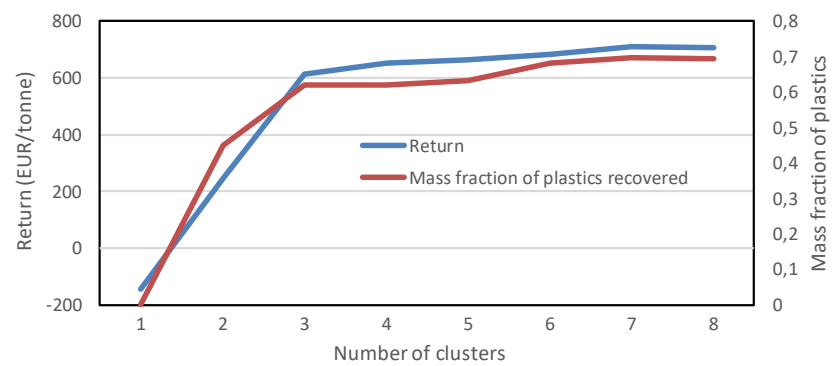

Figure 5. Anticipated return of plastics recycling per tonne of WEEE and recyclable fraction as function of number of clusters for the case study products

The results for the k-Means procedure with three clusters are included in Fig. 4 (rightmost column). The resulting density spectra for the contained plastics per cluster are documented in Fig. 6. In these figures the density separation steps required for post-processing the plastics fraction are marked with vertical lines.

Cluster 0 contains a subset of products and components for which the plastics fraction cannot be retrieved based on density separation only without significant risk for POPs contamination or for blending incompatible polymers. Valorisation of this fraction could still be considered by means of additional sensor based sorting, e.g using LIBS or XRF, or by means of electrostatic separation or froth-flotation techniques $[10,13]$. Such a hybrid procedure is however outside the scope of this study.

\section{Discussion}

The subgraph determination was conducted for a generic list of considered PCR plastics categories and can be used for optimisation exercises with different product sets. Some of the resulting classes are poorly represented in the chosen case study product set (Fig. 4). In order to systematically cover a more complete WEEE stream, the product set should be expanded and documented in terms of composing plastics. Also for the listed product categories a more extensive sampling is targeted: In the framework of the Horizon 2020 PolyCE project such an enhanced data collection effort is being conducted.

It should be noted that the product composition may differ between regions and may evolve over time: the material sampling for the case study data set was conducted in Italian and Belgian WEEE recycling centres and the limited data summarized in Table 1 and Figures 1 and 4 are indicative for products returned in the 2014-2019 period only.

The method can easily take into account components separated from EoL products as part of pre-treatment procedures. Such is, for example, increasingly the case for monitor and TV back covers as part of PCB recovery efforts. 


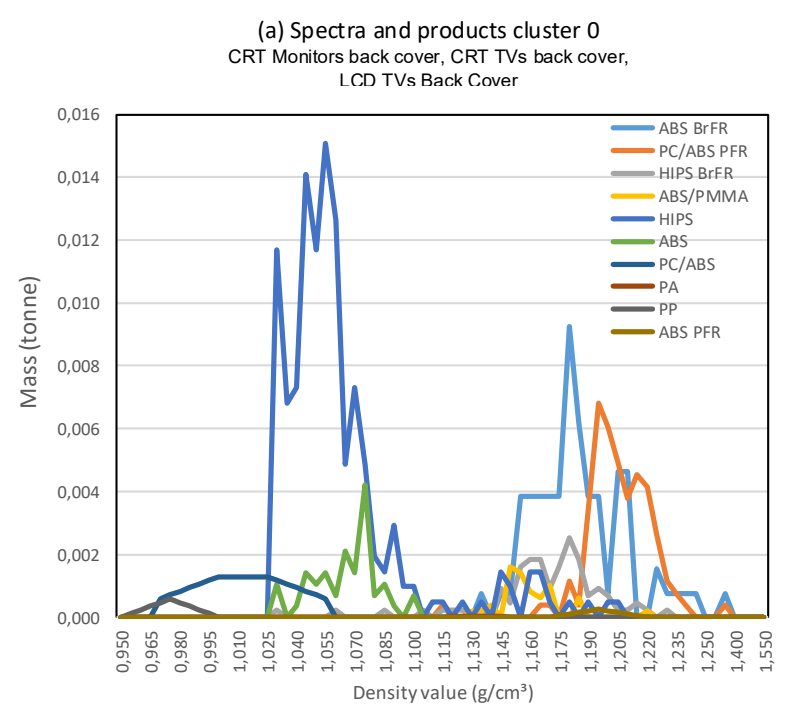

(b) Spectra and products cluster 1 Dishwashers front panel, LCD Monitors back cover, Washing machines tub Washing machines front cover

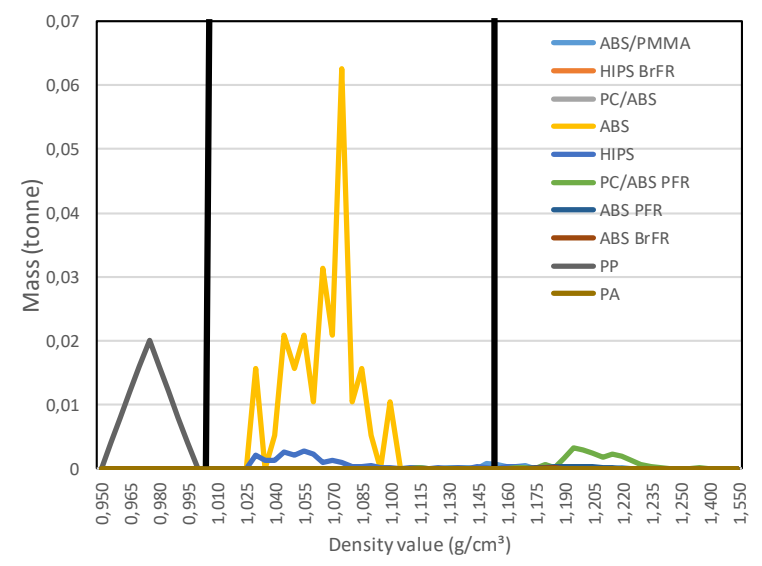

(c) Spectra and products cluster 2 Freezers interior, Refrigerators drawers, Refrigerators door, Refrigerators interior

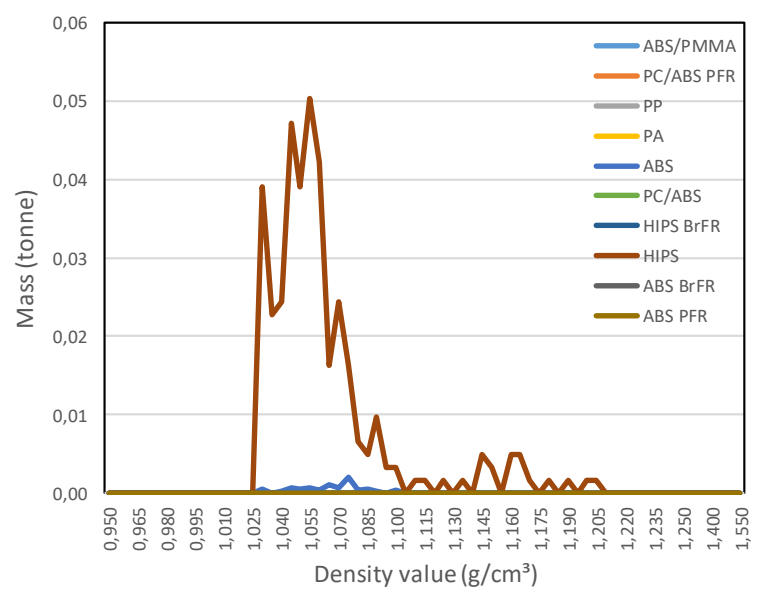

Figure 6. Density spectra for k-Means clustering with $\mathrm{k}=3$ for the product data set as summarized in Table 1 and Fig. 1. The black vertical lines in (b) mark the density based separation limits as required for this cluster.

The described method has been implemented for density based sorting, but can also be extended if sensing systems allowing quick material identification can be called upon for sorting after shredding. Determining an adjusted graph representation matrix allows to take such system capabilities into account.

\section{Conclusions}

When relying on density based separation methods only avoidance of contamination by non-miscible or not ROHS/REACH compliant plastics requires a conservative approach in which a substantial fraction of plastics cannot be recycled due to overlapping density spectra. WEEE product sorting based on the statistically determined polymer content can allow to avoid such separation problems to a large extent. By the clustering of product categories significant increases in the rate of recovered plastic can be achieved, as it avoids the mixing of large amounts of non-compatible plastics with overlapping densities.

The method is generic and can also be used for more refined colour based sorting. It can easily be extended to cover innovative sensor based sorting techniques if statistical data are available documenting their separation capabilities and the processing costs for the considered sensor systems. Given the importance of representative statistical compositional data for the pre-sorting methodology, extensive material sampling efforts are required. Data accessibility is a crucial pre-requisite for the implementation of the presented approach. Continued sampling efforts and collaborative data collection initiatives, also with respect to involved costs, are part of ongoing efforts in this respect.

\section{Acknowledgements}

The authors want to acknowledge the H2020-EU.3.5.4 PolyCE project and the contributions of L. Campadello and $\mathrm{N}$. Vincenti to the data collection efforts underlying the case study.

\section{References}

[1] Baldé C.P., Forti V., Gray V., Kuehr R., Stegmann P., 2017, The Global Ewaste Monitor, United Nations University, International Telecommunication Union \& International Solid Waste Association, Bonn/Geneva/Vienna

[2] Buekens A., Yang J., 2014, Recycling of WEEE plastics: a review, Journal of Material Cycles and Waste Management 16, 415-434

[3] Nnorom, I.C., Osibanjo, O., 2008. Sound management of brominated flame retarded plastics from electronic wastes, Resources, Conservation and Recycling 52, 1362-1372

[4] Schlummer, M., Mäurer, A., 2006. Recycling of styrene polymers from shredded screen housings containing brominated flame retardants. J. Appl. Polym. Sci. 102, 1262-1273

[5] Directive 2012/19/EU of the European Parliament and of the Council of 4 July 2012 on Waste Electrical and Electronic Equipment (recast)

[6] Plastics Europe, 2018, Plastics-The Facts 2018, on-line document, https://www.plasticseurope.org/en/resources/publications/619-plastics-facts2018, last accessed 17/9/2019.

[7] Regulation 2019/1021/EU of the European Parliament and of the Council of 20 June 2019 on Persistent Organic Pollutants (recast)

[8] WRAP, 2009, Separation of Mixed WEEE Plastics, Final Report Project MDD018 and MDD023, Waste \& Resource Action Programme, Banbury, Oxfordshire UK, Report prepared by Axion Consulting

[9] Dewulf W., Wagner F., Bracquené E.,. Peeters J.R, Duflou J.R., 2019, Diversified recycling strategies for high-end plastics: Technical feasibility and impact assessment, CIRP Annals 68/1. 29-32

[10] Peeters, J.R., Vanegas, P. Tange, L., Van Houwelingen, J., Duflou, J.R., 2014, Closed Loop Recycling of Plastics Containing Flame Retardants. Resources, Conservation and Recycling 84, 35-43

[11] Peeters, J.R., Vanegas, P., et al., 2015. Forecasting Waste Compositions: A case study on plastic waste of electronic display housings. Waste Management 46, 28-39

[12] Wilson, R.J., 2010, Introduction to Graph Theory. Harlow, England: Prentice Hall.

[13] Wagner F., Peeters J., De Keyzer J., Duflou J., Dewulf W., 2019, Quality Assessment of Plastic Recyclates from WEEE, Technologies and Ecoinnovation towards Sustainability II, 139-154 